OPEN ACCESS

Edited by:

Nicolau Beckmann

Novartis Institutes for BioMedical

Research, Switzerland

Reviewed by:

Junichi Sadoshima,

University of Medicine and Dentistry

of New Jersey, United States

Martin Landsberger,

Universitätsmedizin Greifswald,

Germany

Yisong Qian,

Nanchang University, China

${ }^{*}$ Correspondence:

Zhuoming L

lizhm5@mail.sysu.edu.cn

Peiqing Liu

liupq@mail.sysu.edu.cn

${ }^{t}$ These authors have contributed

equally to this work

Specialty section:

This article was submitted to

Translational Pharmacology,

a section of the journal

Frontiers in Pharmacology

Received: 25 July 2018

Accepted: 11 December 2018

Published: 08 January 2019

Citation:

Li Z, Zhang X, Guo Z, Zhong Y,

Wang P, Li J, Li Z and LiU P (2019)

SIRT6 Suppresses NFATC4

Expression and Activation

in Cardiomyocyte Hypertrophy.

Front. Pharmacol. 9:1519.

doi: 10.3389/fphar.2018.01519

\section{SIRT6 Suppresses NFATc4 Expression and Activation in Cardiomyocyte Hypertrophy}

\author{
Zhenzhen $\mathrm{Li}^{1,2,3+}$, Xiaoying Zhang ${ }^{4 \dagger}$, Zhen Guo ${ }^{1,2,3}$, Yao Zhong 1,2,3,5, Panxia Wang ${ }^{1,2,3}$, \\ Jingyan $\mathrm{Li}^{1,2,3}$, Zhuoming $\mathrm{Li}^{1,2,3 *}$ and Peiqing Liu ${ }^{1,2,3 *}$
}

\begin{abstract}
'Department of Pharmacology and Toxicology, School of Pharmaceutical Sciences, Sun Yat-sen University, Guangzhou, China, ${ }^{2}$ National and Local United Engineering Lab of Druggability and New Drugs Evaluation, School of Pharmaceutical Sciences, Sun Yat-sen University, Guangzhou, China, ${ }^{3}$ Guangdong Provincial Key Laboratory of New Drug Design and Evaluation, School of Pharmaceutical Sciences, Sun Yat-sen University, Guangzhou, China, ${ }^{4}$ Department of Pharmacology, School of Medicine, Xizang Minzu University, Shaanxi, China, ${ }^{5}$ Department of Cardiology, Third People's Hospital of Dongguan, Dongguan, China
\end{abstract}

NFATc4, a member from the Nuclear Factor of Activated T cells (NFATs) transcription factor family, plays a pivotal role in the development of cardiac hypertrophy. NFATc4 is dephosphorylated by calcineurin and translocated from the cytoplasm to the nucleus to regulate the expression of hypertrophic genes, like brain natriuretic polypeptide (BNP). The present study identified SIRT6, an important subtype of NAD+ dependent class III histone deacetylase, to be a negative regulator of NFATc4 in cardiomyocyte hypertrophy. In phenylephrine (PE)-induced hypertrophic cardiomyocyte model, overexpression of SIRT6 by adenovirus infection or by plasmid transfection repressed the protein and mRNA expressions of NFATc4, elevated its phosphorylation level, prevented its nuclear accumulation, subsequently suppressed its transcriptional activity and downregulated its target gene BNP. By contrast, mutant of SIRT6 without deacetylase activity $(\mathrm{H} 133 \mathrm{Y})$ did not demonstrate these effects, suggesting that the inhibitory effect of SIRT6 on NFATc4 was dependent on its deacetylase activity. Moreover, the effect of SIRT6 overexpression on repressing BNP expression was reversed by NFATc4 replenishment, whereas the effect of SIRT6 deficiency on upregulating BNP was recovered by NFATc4 silencing. Mechanistically, interactions between SIRT6 and NFATc4 might possibly facilitate the deacetylation of NFATc4 by SIRT6, thereby preventing the activation of NFATc4. In conclusion, the present study reveals that SIRT6 suppresses the expression and activation of NFATc4. These findings provide more evidences of the anti-hypertrophic effect of SIRT6 and suggest SIRT6 as a potential therapeutic target for cardiac hypertrophy.

\section{Keywords: cardiomyocyte hypertrophy, SIRT6, deacetylase activity, NFATc4, BNP}

Abbreviations: AAC, abdominal aortic constriction; BNP, brain natriuretic polypeptide; $\mathrm{BW}$, body weight; $\mathrm{CaN}$, calcineurin; $\mathrm{EF}$, ejection fraction; FS, fractional shortening; HE, hematoxylin-eosin; HW, heart weight; LVAW, left ventricular anterior wall thickness; LVID, left ventricular internal diameter; LVPW, left ventricular posterior wall thickness; NC, negative control; NFATc4, nuclear factor of activated T cells c4; NRCMs, neonatal rat cardiomyocytes; NS, normal saline; PBS, phosphate buffer solution; PE, phenylephrine; SD, Sprague-Dawley; siRNA, small-interfering RNA; SIRT6, Sirtuin 6. 


\section{INTRODUCTION}

Cardiac hypertrophy is an adaptive response of the heart to various physiological or pathological stimuli. It is initially a compensatory mechanism to maintain cardiac output. However, prolonged hypertrophy ultimately progresses to arrhythmia, heart failure, or sudden death (Frey et al., 2004; Rohini et al., 2010). Cardiac hypertrophy is characterized by an increase in cell size and protein synthesis, an enhancement of sarcomeric organization, and reactivation of fetal genes, including atrial natriuretic peptide (ANF) and brain natriuretic peptide (BNP), and $\beta$-myosin heavy chain ( $\beta$-MHC) (Cotecchia et al., 2015). A number of complex prohypertrophy signaling cascades have been identified to be involved in the regulation of cardiac hypertrophic response, whereas potential antihypertrophy signaling pathways have been discovered as well, comprising a complex signaling network. Identifying the crosstalk between these pro-hypertrophic and anti-hypertrophic signals helps to understand the molecular and cellular mechanisms underlying the development of cardiac hypertrophy and heart failure (Govindsamy et al., 2018). Augmenting the inhibitory signaling pathways might suggest a promising strategy for treatment of cardiac hypertrophy.

NFATc4, a member of nuclear factor of activated $\mathrm{T}$ cells (NFAT) transcription factor family, has been well-documented for its involvement in several diseases including cardiac hypertrophy, by regulating various target genes (Horsley and Pavlath, 2002; Li et al., 2016a, 2017; Zhang and Storey, 2016; Wang et al., 2017; Sharma et al., 2018). Similar as other NFAT subtypes, NFATc4 is dephosphorylated and activated by $\mathrm{CaN}$ (van Middendorp et al., 2017). After dephosphorylation, NFATc4 is transported from the cytoplasm to the nucleus and binds to target DNA to regulate the expression of hypertrophic genes, in particular the hypertrophic marker BNP (Molkentin et al., 1998; Crabtree, 1999; Liu et al., 2015; Li et al., 2016a, 2017). In addition, NFATc4 interacts with other hypertrophic signaling pathways, such as GATA-4, MAPK, GSK3, p38, and JNK (Coleman et al., 2015). Thus, CaN-NFAT signaling pathway has been regarded as a critical pathway that induces cardiac hypertrophy (Molkentin et al., 1998; Zhao et al., 2016).

Sirtuins, first discovered in yeast as silent information regulator 2 (Sir2), catalyzes $\mathrm{NAD}^{+}$-dependent histone and non-histone deacetylation and thus participates in many pathophysiological processes (Heineke and Molkentin, 2006; Sosnowska et al., 2017; Tasselli et al., 2017). The family of sirtuins contains seven subunits in mammals (SIRT1-SIRT7) that share a conserved core catalytic domain but differ in their cellular localization and tissue distribution. Among all, the nucleardistributed SIRT6 has recently attracted numerous attentions for its critical role in regulating gene stability, metabolism, stress response, and aging (Mendes et al., 2017). Previous studies in our laboratory and others have revealed that SIRT6 can prevent and inhibit cardiac hypertrophy (Sundaresan et al., 2012; Yu et al., 2013; Shen et al., 2016; Zhang et al., 2016). SIRT6 suppresses cardiomyocyte hypertrophy via interaction with nuclear factor kappa B (NF-кB) catalytic subunit p65 to inhibit its transcriptional activity (Yu et al., 2013), via interaction with c-Jun and deacetylation of histone 3 at Lys9 (H3K9) to repress insulin-like growth factor signaling (Sundaresan et al., 2012), via repression of STAT3 (signal transducer and activator of transcription 3) (Zhang et al., 2016), and via decreasing the acetylase p300 expression by promoting its degradation (Shen et al., 2016). All these effects of SIRT6 are dependent on its deacetylase activity. The decrease of SIRT6 deacetylase activity in response to pressure overload or hypertrophic stimuli such as angiotensin II, isoprenaline and PE contributes to the pathogenesis of cardiac hypertrophy, although its expression is not altered (Cai et al., 2012; Yu et al., 2013; Lu et al., 2016; Shen et al., 2016; Zhang et al., 2016).

In this study, we identify that SIRT6 could suppress CaNNFAT signaling pathway in cardiomyocyte hypertrophy. This mechanism provides a novel insight into the anti-hypertrophic effect of SIRT6 and suggests SIRT6 as a potential therapeutic target for cardiac hypertrophy.

\section{MATERIALS AND METHODS}

\section{Antibodies and Reagents}

Anti-NFATc4 (diluted 1:200, sc-13036), anti-phospho-NFATc4 (diluted 1:100, sc-32630) polyclonal antibodies were purchased from Santa Cruz Biotechnology Inc. (Santa Cruz, CA, United States). Anti-SIRT6 (diluted 1:1000, 12486) antibody was bought from Cell Signaling Technology (Beverly, MA, United States). Anti-CaN A (diluted 1:1000, ab90540) antibody was purchased from Abcam (Cambridge, MA, United States). Anti-p38 (diluted 1:1000, 14064-1-AP) antibody was bought from Proteintech (Rosemont, IL, United States). Anti- $\alpha$-tubulin (diluted 1:1000, T9026) and anti-Lamin B1 (diluted 1:1000, SAB1306342) antibodies were obtained from Sigma (St. Louis, MO, United States). Lipofectamine 2000 was obtained from Invitrogen (Carlsbad, CA, United States). PE was obtained from Tocris Bioscience (Bristol, United Kingdom). Rhodamine phalloidin and 4'6-diamidino-2-phenylindole (DAPI) were purchased from Invitrogen (Carlsbad, CA, United States). Other reagents were from Sigma-Aldrich unless otherwise stated.

\section{Primary Culture of Neonatal Rat Cardiomyocytes}

The primary culture of NRCMs were prepared as previously described ( $\mathrm{Yu}$ et al., 2013). NRCMs were isolated from the ventricles of 1 to 3-day-old SD rats. Briefly, hearts were removed immediately and kept in cold phosphate-buffered saline (PBS), and the minced ventricles were dispersed at $37^{\circ} \mathrm{C}$ in $0.08 \%$ trypsin solution approximately $12-14$ times for $5 \mathrm{~min}$ each time. Cell suspension from each digestion was gathered. Finally, the cells were harvested by centrifugation for $8 \mathrm{~min}$ at $1400 \times g$ and then suspended in Dulbecco's modified Eagle's medium (DMEM, Gibco, BRL Co., Ltd., United States) supplemented with $10 \%$ fetal bovine serum (FBS). The suspensions were plated in culture flasks for $1 \mathrm{~h}$ at $37^{\circ} \mathrm{C}$ in a humidified atmosphere $(5 \%$ $\mathrm{CO}_{2}$ and 95\% air). NRCMs were seeded onto culture dishes in DMEM supplemented with 10\% FBS and 5-bromodeoxyuridine (0.1 mM). PE, a $\alpha 1$ adrenergic receptor agonist, is commonly 
used to induce cardiomyocyte hypertrophy (Appert-Collin et al., 2007). The NRCMs were treated with $100 \mathrm{mM}$ PE for 6,12 , or $24 \mathrm{~h}$ to induce hypertrophy.

\section{Animal Model, Chocardiography, and Morphometric Measurements}

Sprague-Dawley rats (male, 200-220 g, SPF grade, Certification No. 44005800006455) were obtained from the Experimental Animal Center of Sun Yat-sen University (Guangzhou, China). The animal experiments were approved by the Research Ethics Committee of Sun Yat-sen University and were in accordance with the Guide for the Care and Use of Laboratory Animals (NIH Publication No 85-23, revised 1996). AAC surgery was conducted as previously described (Yu et al., 2013). Briefly, rats were randomly divided into two groups (the Sham group and the AAC group) and anesthetized with $10 \%$ chloral hydrate $(350 \mathrm{mg} / \mathrm{kg}$, ip). The adequacy of anesthesia was monitored by evaluating and recording body temperature, cardiac and respiratory rates and patterns, muscle relaxation, and lash reflex. Under sterile conditions, the abdominal aorta above the kidneys was exposed through a midline abdominal incision and constricted at $4-5 \mathrm{~cm}$ above the suprarenal artery with a 5-0 silk suture that was tied around both the aorta and a blunted 22-gauge needle. The needle was promptly removed after constriction. The Sham group underwent a similar procedure without banding the aorta. After the operation, the surgical wound was sutured, and gentamicin was given to prevent infection. Eight weeks after AAC surgery, twodimensional-guided $\mathrm{M}$-mode echocardiography was performed by a Technos MPX ultrasound system (ESAOTE, SpAESAOTE SpA, Italy) (Zhou et al., 2006). Afterward, the rats were sacrificed and then their hearts were quickly removed for trimming the left ventricles. $5-\mu \mathrm{m}$-thick histological cross sections of the heart tissues were stained with HE for morphometric measurement.

\section{Western Blot Analysis}

Western blotting was performed as previously described (Guo et al., 2015). Proteins of cultured cells or rat left ventricular tissues were harvested using RIPA lysis buffer (Beyotime Nantong, Jiangsu, China) containing a protease inhibitor cocktail (Sigma-Aldrich, St. Louis, MO, United States) on ice. Nuclear extracts were prepared using the CelLytic Nuclear Extraction Kit (Sigma-Aldrich, St. Louis, MO, United States), according to the manufacturer's instructions. Protein concentrations were measured using a bicinchoninic acid (BCA) Protein Assay Kit (Pierce, Rockford, IL, United States). Equal amount of proteins $(35 \mu \mathrm{g}$ of total proteins or $15 \mu \mathrm{g}$ of nuclear proteins) were separated by $8 \sim 12 \%$ sodium dodecyl sulfatepolyacrylamide gel electrophoresis (SDS-PAGE) and then transferred to polyvinylidene fluoride (PVDF) membranes (EMD Millipore Corporation, Billerica, MA, United States). The membranes were incubated with primary antibodies over night at $4{ }^{\circ} \mathrm{C}$, followed by incubating with appropriate horseradish peroxidase-conjugated secondary antibodies at room temperature for $1 \mathrm{~h}$. Blots were developed with enhanced chemiluminescence reagent (Pierce, Rockford, IL, United States) and detected by the LAS4000 imager (GE Healthcare, Waukesha, WI, United States). The intensities of the blots were quantified by the Quantity One (Bio-Rad) software. $\alpha$-tubulin was used as an internal control for total proteins, and Lamin B1 was used for normalizing nuclear proteins.

\section{RNA Extraction and Quantitative Real-Time Polymerase Chain Reaction (qRT-PCR)}

Total RNA from heart tissues or cultured cells was extracted using Trizol Reagent (Invitrogen, Carlsbad, CA, United States) following the manufacturer's instruction. A total of $5 \mu \mathrm{g}$ RNA were reversely transcribed to first strand cDNA using two-step RT kit (Thermo, Fisher Scientific, Rockford, IL, United States). The mRNA levels of the target genes were determined using the SYBR Green Quantitative PCR kit (TOYOBO, Japan) on the Real-time Thermo Cycler (Thermo Fisher Scientific, Rockford, IL, United States) as previously described (Guo et al., 2018). Rat-specific primer for miR-133a and miR-29a-3p were purchased from Ribobio Co., Ltd. (Guangzhou, China). Ratspecific primers for BNP, SIRT6, NFATc4, and $\beta$-actin (listed in Supplementary Table S1) were synthesized by Sangon Biotech Co., Ltd. (Shanghai, China). U6 and $\beta$-actin were used as an internal control.

\section{Measurement of Cell Surface Area}

NRCMs cultured in 48-well plates were fixed with $4 \%(w / v)$ paraformaldehyde in phosphate-buffered saline (PBS) for $15 \mathrm{~min}$ at room temperature or overnight at $4{ }^{\circ} \mathrm{C}$, followed by $0.5 \%$ Triton-100 treatment for $10 \mathrm{~min}$. Then the cell incubation with $0.1 \%(\mathrm{v} / \mathrm{v})$ rhodamine-phalloidin for $30 \mathrm{~min}$, were washed with PBS three times and counterstained with DAPI. Images of the NRCMs were detected by the High Content Screening system (ArrayScanVTI, Thermo Fisher Scientific, Rockford, IL, United States). The cell surface area from randomly selected fields (50 for each group) was determined using the built-in image analysis software.

\section{Adenovirus Vector}

Recombinant adenovirus vectors expressing green fluorescent protein (Ad-GFP) and GFP-tagged SIRT6 (Ad-SIRT6), respectively, were purchased from Hanbio Technology (Shanghai, China). The viruses were expanded in HEK293A cells and purified with a virus purification kit (Biomiga, United States). The purified viruses were dialyzed in dilution buffer and stored at $-80^{\circ} \mathrm{C}$.

\section{Plasmid Transfection}

Rat-SIRT6 (NM_001031649.1) gene was cloned into the pcDNA3.1+ vector by clone site HindIII/XhoI with flag tag and confirmed by DNA sequencing in Sangon Biotech Co., Ltd. (Shanghai, China). SIRT6 mutant (H133Y) was constructed using the Fast Mutagenesis System (TRANS, Beijing, China) according to the manufacturer's instructions. The following 
primers were used for mutagenesis: forward, 5'-GGA CAA GCT GGC CGA GCT GTA CGG AAA CAT-5' ; reverse, 5' -ACA GCT CGG CCA GCT TGT CCC TGG GGA A-3'. NRCMs were transiently transfected with $4 \mu \mathrm{g}$ WT-SIRT6 and H133Y using $5 \mu \mathrm{L}$ Lipofectamine 2000 (Invitrogen Carlsbad, CA, United States) according to the manufacturer's instructions.

\section{RNA Interference}

Small inference RNA (siRNA) targeting SIRT6, NFATc4, and NC siRNA were obtained from Genepharma (Shanghai, China). The sequences of the siRNAs are shown in Supplementary Table S2. NRCMs seeded in $35 \mathrm{~mm}$ dishes were transfected with $100 \mathrm{pmol}$ of targeted siRNA or NC siRNA using $5 \mu \mathrm{L}$ of Lipofectamine 2000 (Invitrogen, Carlsbad, CA, United States) according to the manufacturer's instructions. NRCMs were transfected with SIRT6 and NFATc4 siRNAs for $48 \mathrm{~h}$. The control groups were transfected with NC sequences.

\section{Immunofluorescence (IF) Assay}

NRCMs seeded on coverslips were fixed with $4 \%$ paraformaldehyde for $10 \mathrm{~min}$. After washing with PBS for three times, the cells were permeabilized with $0.3 \%$ TritonX-100 for $5 \mathrm{~min}$ and followed by incubation with blocking solution at room temperature for $1 \mathrm{~h}$. The cells were further treated for $1 \mathrm{~h}$ with primary antibody against NFATc4 (diluted 1:50), and incubated with Alexa Fluor-labeled secondary antibody (diluted 1:200, Santa Cruz Biotechnology, United States). The coverslips were mounted with DAPI and were detected by a confocal microscope (LSM 710, Carl Zeiss, Jena, Germany).

\section{Luciferase Reporter Gene Assay}

NFATc4 reporter gene plasmid (inserted sequence of AGCAAC) was constructed with pGL3-Basic and confirmed by DNA sequencing in Sangon Biotech Co., Ltd. (Shanghai, China). The promega Renilla Luciferase vector contained herpes simplex virus thymidine kinase promoter (pRL-TK) and NFATc4 reporter plasmids pGL3. Dual-luciferase reporter assay system were obtained from Promega (Madison, WI, United States). Cells were seeded at $1 \times 10^{5}$ cells.well ${ }^{-1}$ into 48 -well plates, and cotransfected with NFATc4 reporter plasmid (400 ng per well) and pRL-TK (80 ng per well) as internal control. Total amounts of transfected DNA were equalized by the addition of empty vector. After $6 \mathrm{~h}$ incubation, the cells were infected with Ad-SIRT6 or Ad-GFP, followed by PE stimulation. Luciferase activity was measured by dual-luciferase reporter assay system (Promega) and normalized by Renilla luciferase activity of pRL-TK.

\section{Immunoprecipitation}

Experiments were performed as previously described. $200 \mu \mathrm{g}$ total protein were incubated with anti-NFATc4 antibody (diluted 1:200) or rat normal IgG (used as a control) overnight at $4^{\circ} \mathrm{C}$ and then incubated with protein G/A agarose beads (Thermo Fisher Scientific, Rockford, IL, United States) for 4 h. Afterward, the beads were washed with lysis buffer for three times and then boiled in loading buffer for $5 \mathrm{~min}$. The immunoprecipitated proteins were detected by Western blotting (Yuan et al., 2014).

\section{Statistical Analysis}

The data are presented as the means \pm SEM. Differences between two groups were analyzed with Student's $t$-test. Statistical analysis among the various groups was conducted by one-way analysis of variance (ANOVA) with Tukey's post hoc test. In all cases, a $P$-value $<0.05$ was considered statistically significant.

\section{RESULTS}

\section{Calcineurin/NFATc4 Signaling Pathway Was Activated in Cardiac Hypertrophy}

The $\alpha$-adrenoceptor agonist PE has been widely used to stimulate cardiomyocyte hypertrophy. In our study, treatment with $100 \mu \mathrm{M}$ PE for $12 \mathrm{~h}$ significantly increased the cell surface area in NRCMs (Figure 1A). The expression of BNP, a marker of cardiac hypertrophy, was enhanced by $100 \mu \mathrm{M}$ PE treated for 6, 12, and $24 \mathrm{~h}$ (Figure 1B). These results suggested that the PE-induced hypertrophic cardiomyocyte model was successfully established. In these hypertrophic cardiomyocytes, the protein expression of NFATc4 was significantly increased, whereas its phosphorylation level at Ser 168 and Ser 170 (pNFATc4) was dramatically decreased (Figure 1C), resulting in a reduction of the $\mathrm{p}-\mathrm{NFATc} 4 / \mathrm{NFATc} 4$ ratio (Figure 1D). In addition, the protein expression of $\mathrm{CaN}$ was augmented by PE treatment (Figure 1E). Since NFATc4 is transported to the nucleus and activated following dephosphorylation by $\mathrm{CaN}$, these observations thus indicate that the CaN/NFATc4 signaling pathway is activated in PE-induced cardiomyocyte hypertrophy.

To further confirm the changes of CaN/NFATc4 signaling pathway in cardiac hypertrophy in vivo, the rat cardiac hypertrophy model was induced by AAC operation. As shown in Figure 2A, the hearts from the AAC rats were larger than those from the Sham group. Pressure overload induced an increase in the myocyte diameter showed by $\mathrm{HE}$ staining (Figure 2B), an enhancement in LVAW and LVPW showed by echocardiography (Figure 2C and Supplementary Table S3), as well as an elevation in both the ratio of $\mathrm{HW} / \mathrm{BW}$ and the protein expression of $\mathrm{BNP}$ (Figures 2D,E). In the hearts of AAC rats, the expressions of NFATc4 and CaN were upregulated, but the phosphorylation level of NFATc4 was attenuated (Figures 2F,H). As a result, the p-NFATc4/NFATc4 ratio was decreased (Figure 2G). These results are consistent with the in vitro observations showing that CaN/NFATc4 is activated during cardiac hypertrophy.

\section{SIRT6 Protected Against Cardiomyocyte Hypertrophy and Suppressed the Expression and Dephosphorylation of NFATc4 Dependent on Its Deacetylase Activity}

To explore the regulatory role of SIRT6 in cardiac hypertrophy and CaN/NFATc4 pathway, NRCMs were infected with adenovirus vector encoding SIRT6 cDNA 
A
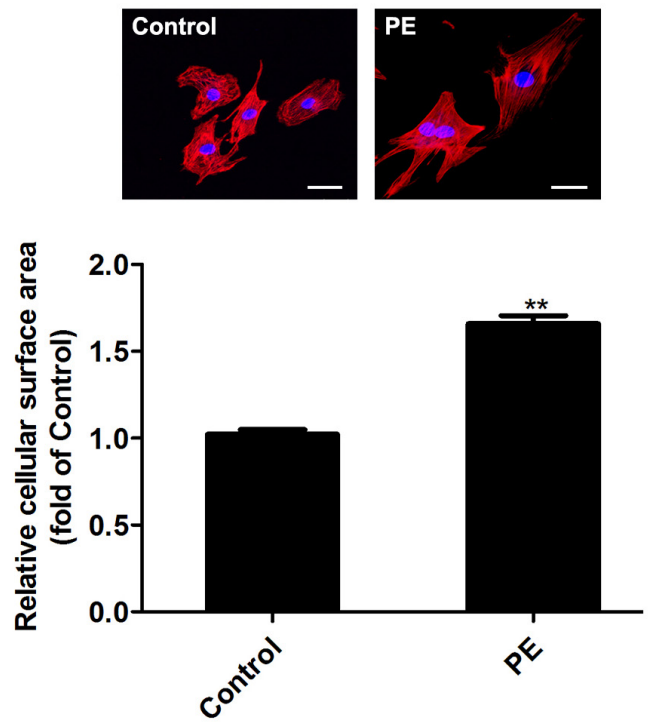

C

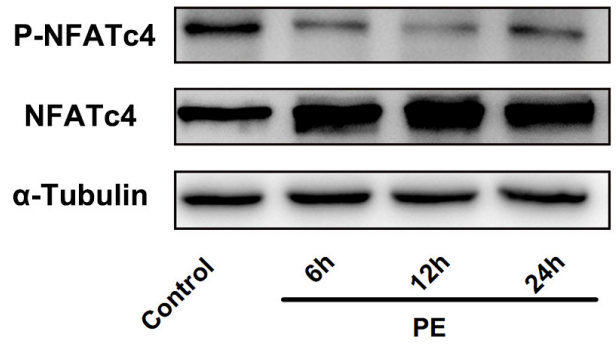

D

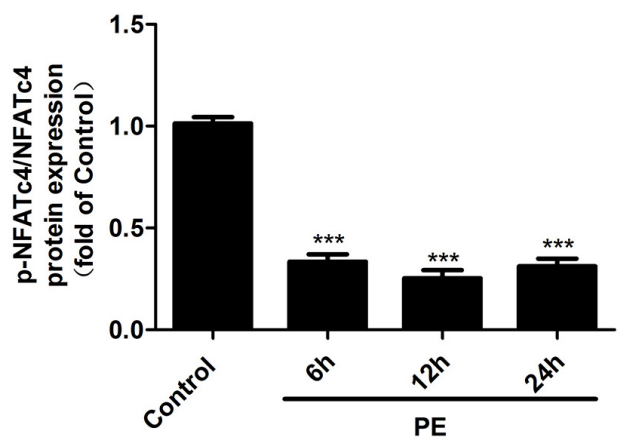

B
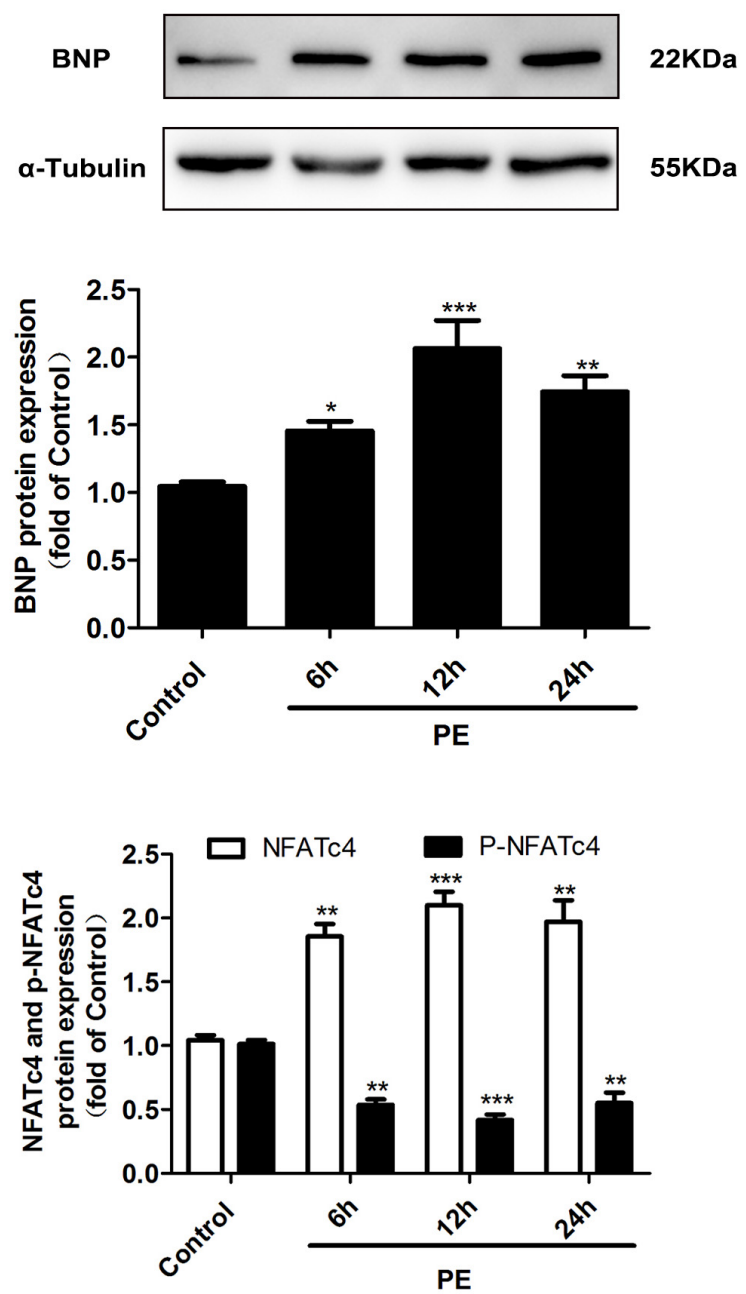

E
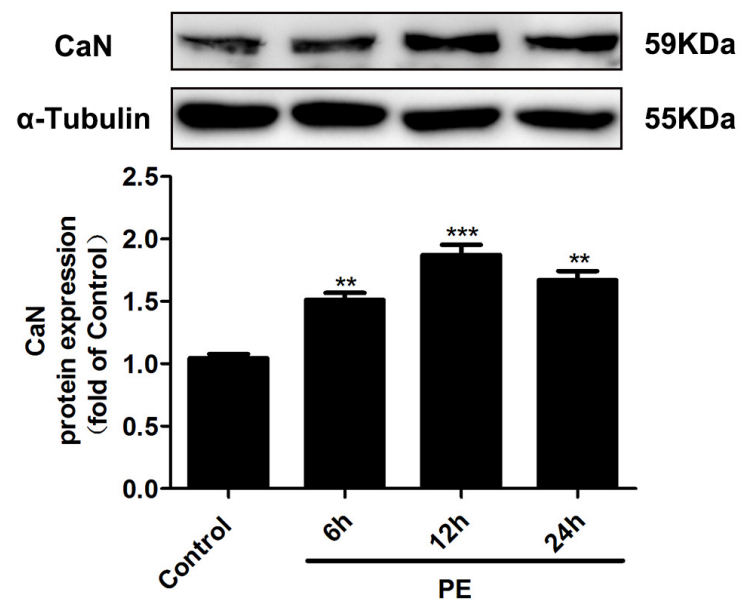

FIGURE 1 | Changes of NFATc4 expression in PE-induced cardiac hypertrophy in vitro. Cultured NRCMs were incubated with $100 \mu \mathrm{M}$ PE for the indicated durations. (A) The cell surface area was measured by rhodamine-phalloidin staining. Scale bar: $20 \mu \mathrm{m}$. Western blotting analysis was conducted to determine the protein expression of (B) BNP, (C) total NFATc4 and phosphorylated NFATc4, (D) p-NFATc4/NFATc4 ratio, and (E) calcineurin in NRCMs treated with $100 \mu \mathrm{M}$ PE. The data were presented as mean \pm SEM. ${ }^{*} P<0.05,{ }^{* *} P<0.01,{ }^{* * *} P<0.001$ vs. Control, $n=4$. 
A

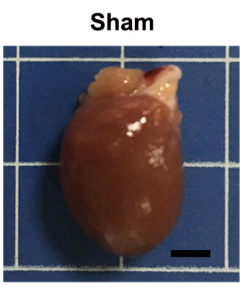

C

Sham
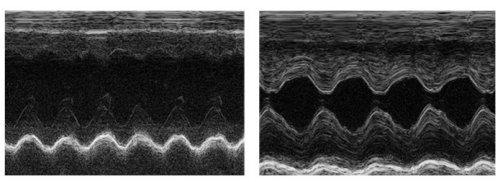

E
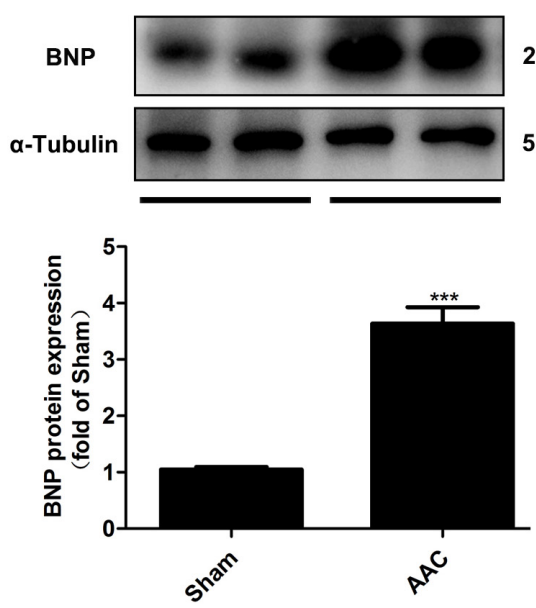

G

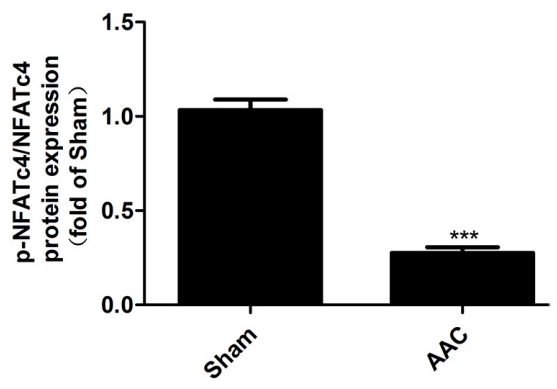

B

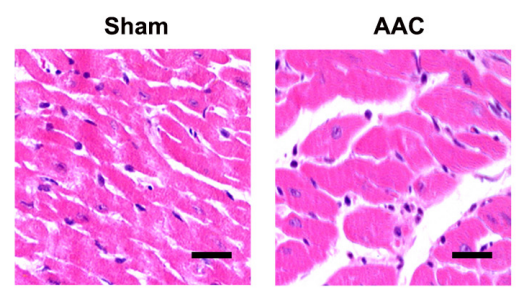

D

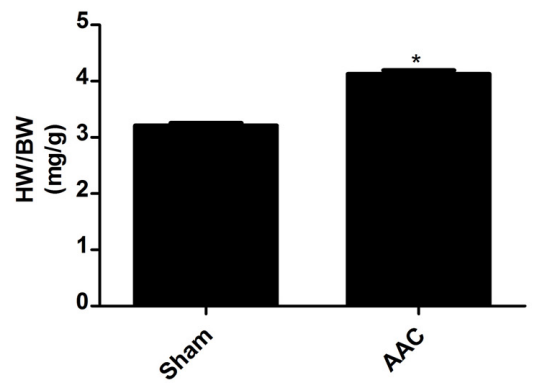

$\mathbf{F}$

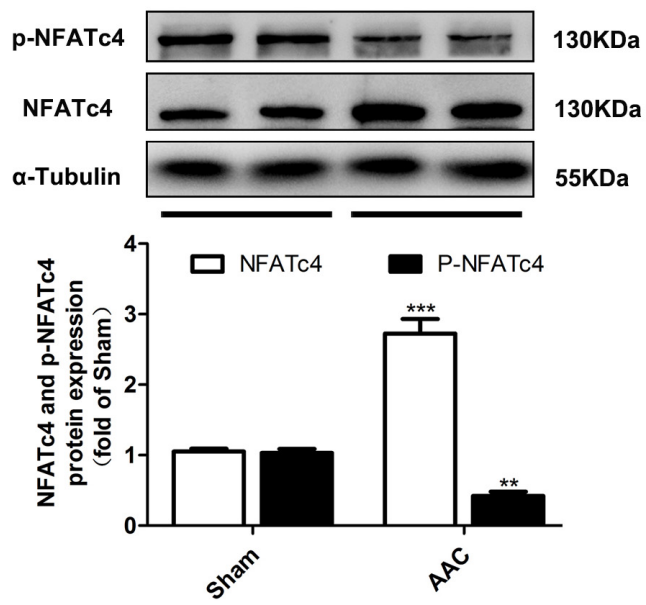

H
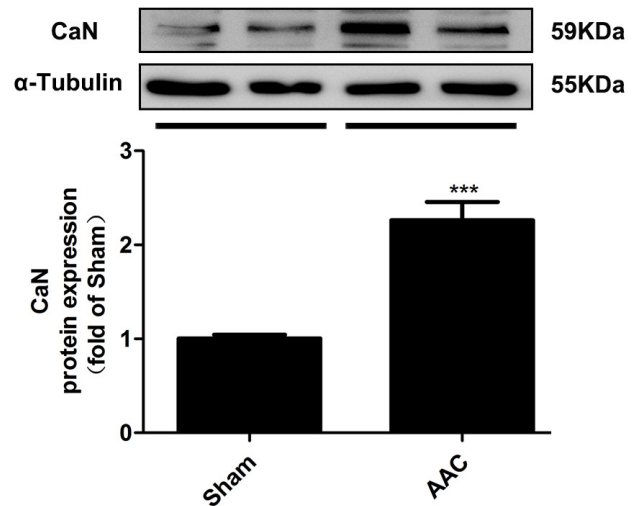

FIGURE 2 | Changes of NFATc4 expression in pressure overload-induced cardiac hypertrophy in vivo. (A) Gross hearts from both AAC and Sham rats. Scale bar: $0.4 \mathrm{~cm}$. (B) Histological analysis detected by hematoxylin and eosin staining. Scale bar: $10 \mu \mathrm{m}$. (C) Representative echocardiography analysis for both AAC and Sham rats. (D) The ratio of heart weight to body weight (HW/BW) were measured. Western blotting analysis was conducted to determine the protein expression of (E) BNP, (F) total NFATc4, phosphorylated NFATc4, (G) p-NFATc4/NFATc4 ratio, and (H) the protein expression of calcineurin in AAC and Sham rats. The data were presented as mean \pm SEM. ${ }^{*} P<0.05,{ }^{* *} P<0.01,{ }^{* * *} P<0.001$ vs. Sham, $n=6$. 


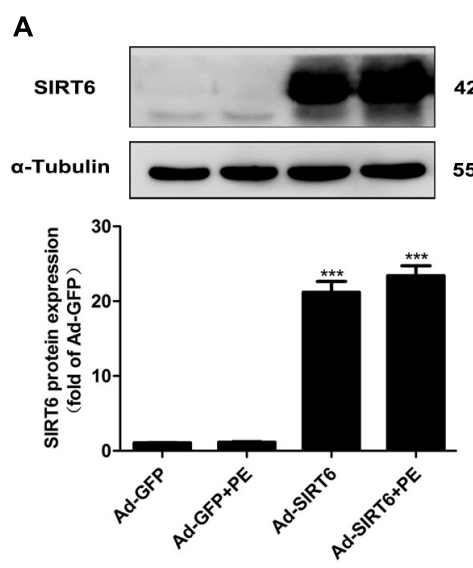

D

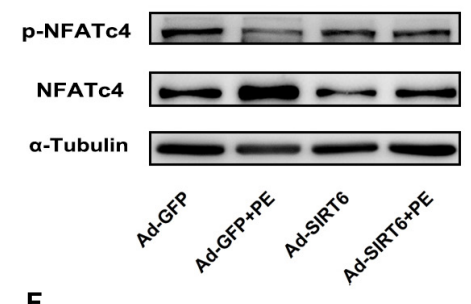

$\mathbf{F}$

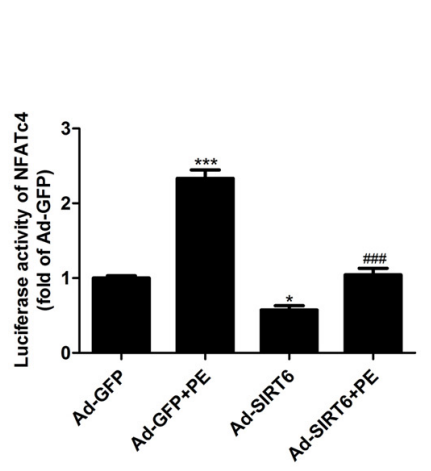

B
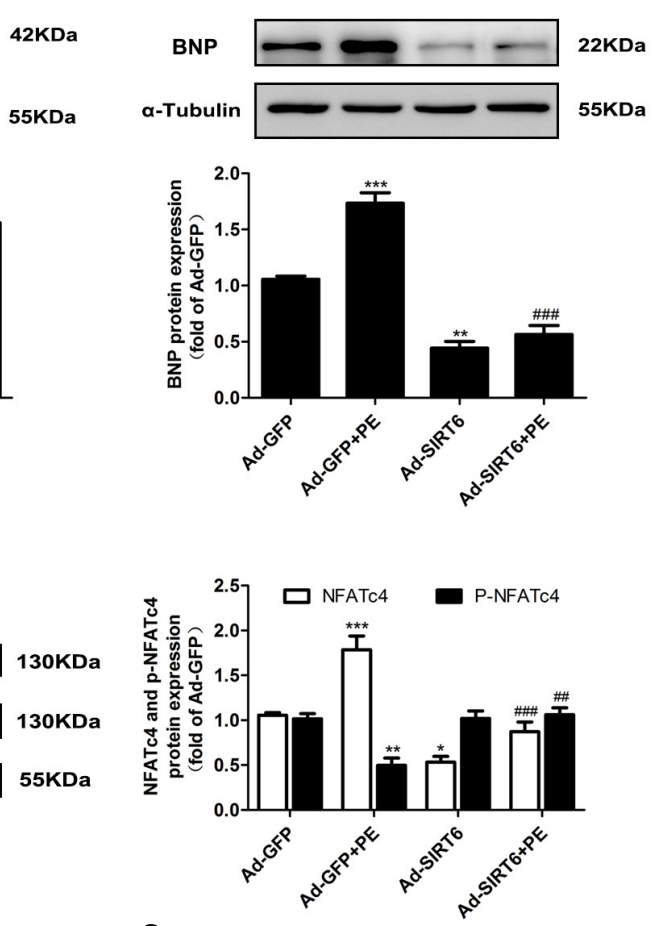

G
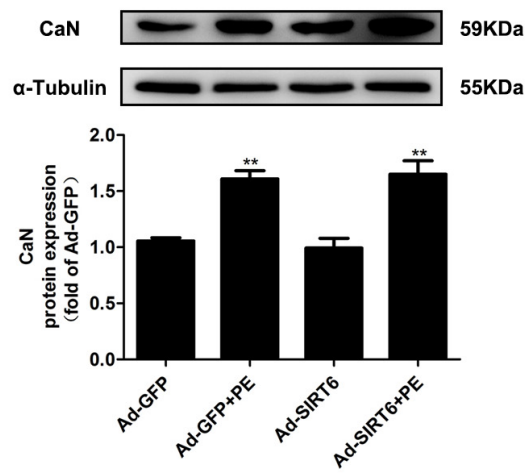

C

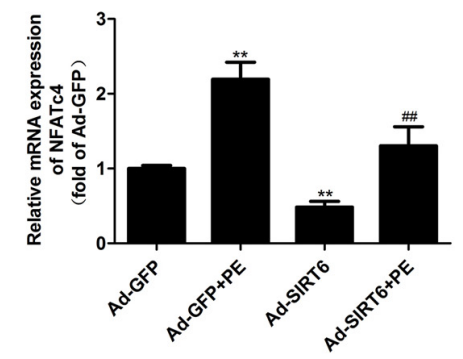

E

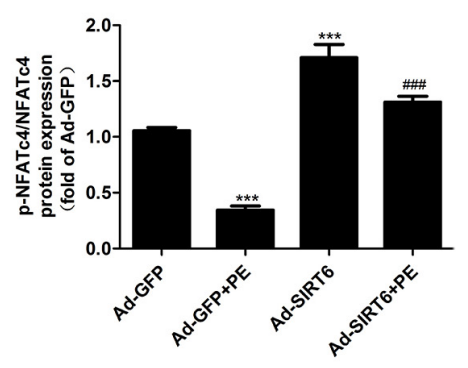

FIGURE 3 | SIRT6 overexpression suppressed the expression level and dephosphorylation of NFATc4. NRCMs were infected with adenovirus vector encoding SIRT6 cDNA for $48 \mathrm{~h}$. (A,B) The protein expression of SIRT6 and BNP were determined by Western blot analysis. (C) The mRNA expression of NFATc4 was determined by qRT-PCR. (D) Total NFATc4 and phosphorylated NFATc4 were investigated by Western blotting. (E) The ratio of p-NFATc4/NFATc4 was determined. (F) Dual luciferase reporter assays evaluating NFATc4-dependent transcriptional activity. (G) The protein expression of calcineurin was studied in NRCMs treated with $100 \mu \mathrm{M}$ PE for $12 \mathrm{~h}$. The data were presented as mean \pm SEM. ${ }^{*} P<0.05,{ }^{* *} P<0.01,{ }^{* * *} P<0.001$ vs. Ad-GFP. ${ }^{\# \#} P<0.01,{ }^{\# \# \#} P<0.001$ vs. Ad-GFP + PE, $n=4$.

(Figure 3A). Ad-SIRT6 infection significantly reduced the protein expression of BNP, suggesting that SIRT6 protects the cardiomyocytes against PE-induced hypertrophy (Figure 3B). Ad-SIRT6 not only attenuated the mRNA and protein expression of NFATc4, but also reversed the decreased level of NFATc4 phosphorylation and the reduction of $\mathrm{p}$-NFATc4/NFATc4 ratio induced by PE treatment (Figures 3C-E). Moreover, the effect of SIRT6 on the transcriptional activity of NFATc4 was evaluated by a dual luciferase reporter gene assay. Ad-SIRT6 repressed the transcriptional activity of NFATc4 in the presence or absence of PE (Figure 3F). However, SIRT6 overexpression did not alter the protein expression of $\mathrm{CaN}$ (Figure 3G).

To further elucidate whether or not the effect of SIRT6 on NFATc4 is dependent on its deacetylase activity, we constructed the plasmids encoding the wildtype SIRT6 (WT-SIRT6) and mutant of SIRT6 without deacetylase activity (H133Y). First, to validate our method, we transfected NRCMs with GFP-SIRT1 plasmid by using Lipofectaminise 2000. The cells were observed under fluorescence microscope, which showed a high transfection efficiency (Supplementary Figure S3A). Consistent with the observations by AdSIRT6 infection, transfection of WT-SIRT6 decreased the 
A

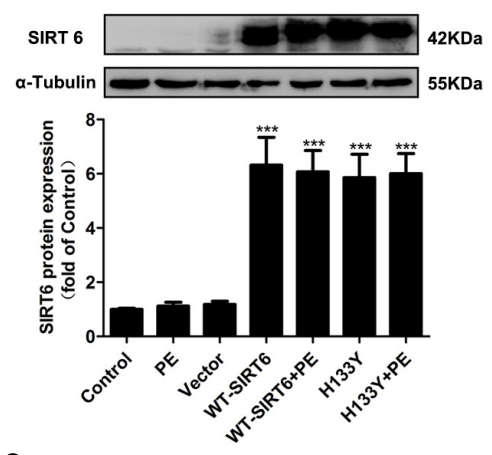

C
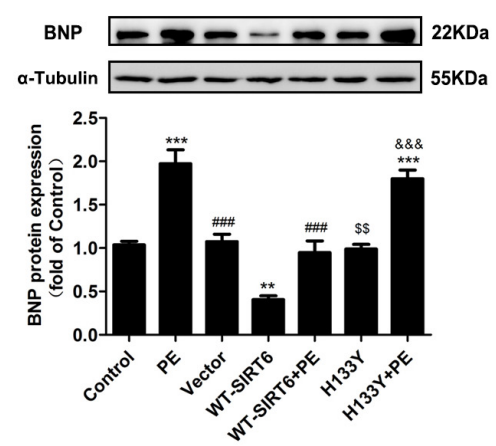

$\mathbf{F}$

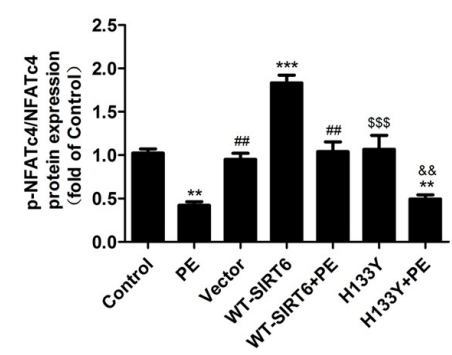

B

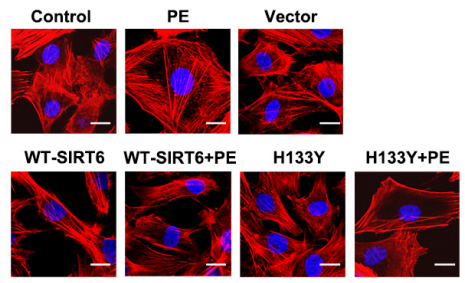

D

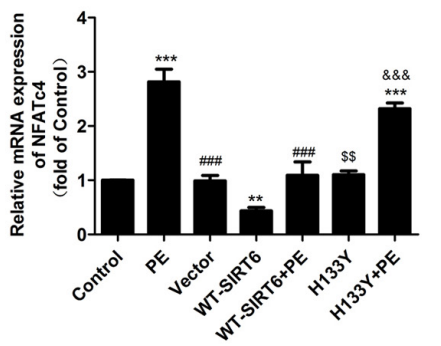

G
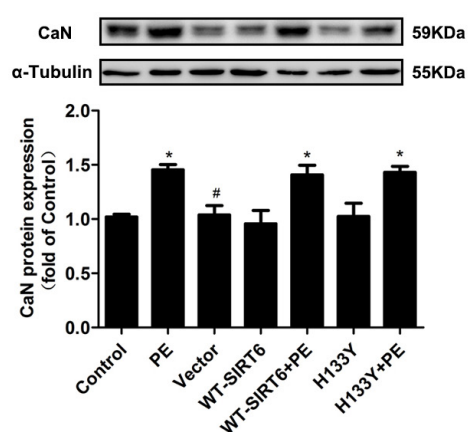

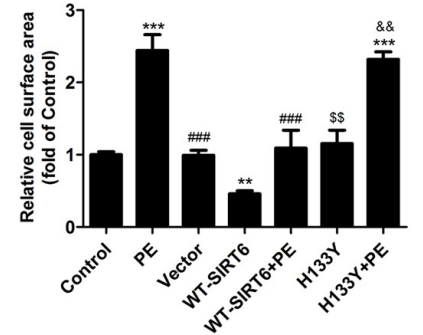

E
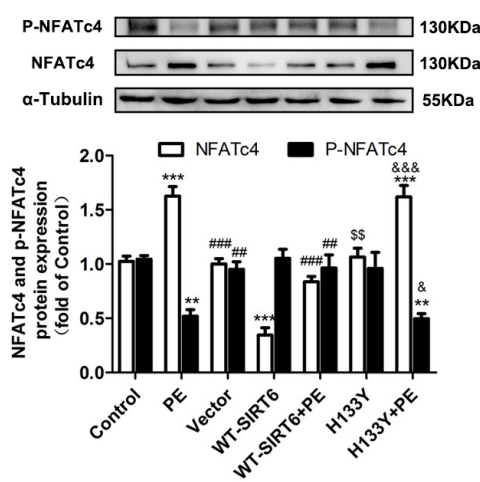

FIGURE 4 | The effects of SIRT6 were dependent on deacetylase activity. NRCMs were transiently transfected with plasmid encoding the WT-SIRT6 and mutant of SIRT6 (H133Y) for $48 \mathrm{~h}$ followed by incubation with PE (100 $\mu \mathrm{M}$ for $12 \mathrm{~h}$ ). (A) Western blot analysis was conducted to determine the protein expression of SIRT6. (B) The cell surface area was measured by rhodamine-phalloidin staining. Scale bar: $20 \mu \mathrm{m}$. (C) Western blot analysis was conducted to determine the protein expressions of BNP. (D) The mRNA expression of NFATc4 was determined by qRT-PCR. (E) The protein expression of total NFATc4, phosphorylated NFATc4 was determined by Western blotting. (F) The ratio of p-NFATc4/NFATc4 was calculated. (G) The protein expression of calcineurin was studied in NRCMs treated with $100 \mu \mathrm{M}$ PE for $12 \mathrm{~h}$. The data were presented as mean \pm SEM. ${ }^{*} P<0.05,{ }^{* *} P<0.01,{ }^{* * *} P<0.001$ vs. Control. ${ }^{\#} P<0.05,{ }^{\# \#} P<0.01,{ }^{\# \# \#} P<0.001$, vs. $P E$. $\$ \$ P<0.01,{ }^{\$} \$ P<0.001$, vs. WT-SIRT6. ${ }^{\&} P<0.05,{ }^{\&} \& P<0.01,{ }^{\& \&} \& P<0.001$, vs. WT-SIRT6 + PE. $n=4$.

cell surface area and BNP expression in NRCMs treated with or without PE (Figures 4A-C), downregulated the mRNA and protein expression of NFATc4 (Figures 4D,E), and recovered the repression of p-NFATc4 level and p-NFATc4/NFATc4 ratio caused by PE treatment (Figures 4E,F), without affecting CaN expression (Figure 4G). Unlike WTSIRT6, transfection with the SIRT6 mutant H133Y did not ameliorate cardiomyocyte hypertrophy (Figures $4 \mathrm{~A}-\mathrm{C}$ ), did not alter NFATc4 expression, the p-NFATc4 level or the pNFATc4/NFATc4 ratio (Figures $4 \mathbf{D}-\mathbf{F}$ ). These results indicate that the inhibitory effect of SIRT6 on NFATc4 expression and dephosphorylation is dependent on its deacetylase activity.

\section{SIRT6 Prevented PE-Induced Nuclear Translocation of NFATc4 Dependent on Its Deacetylase Activity}

The activation of NFATc4 requires dephosphorylation at Ser 168 and Ser 170 and subsequent translocation from 
A

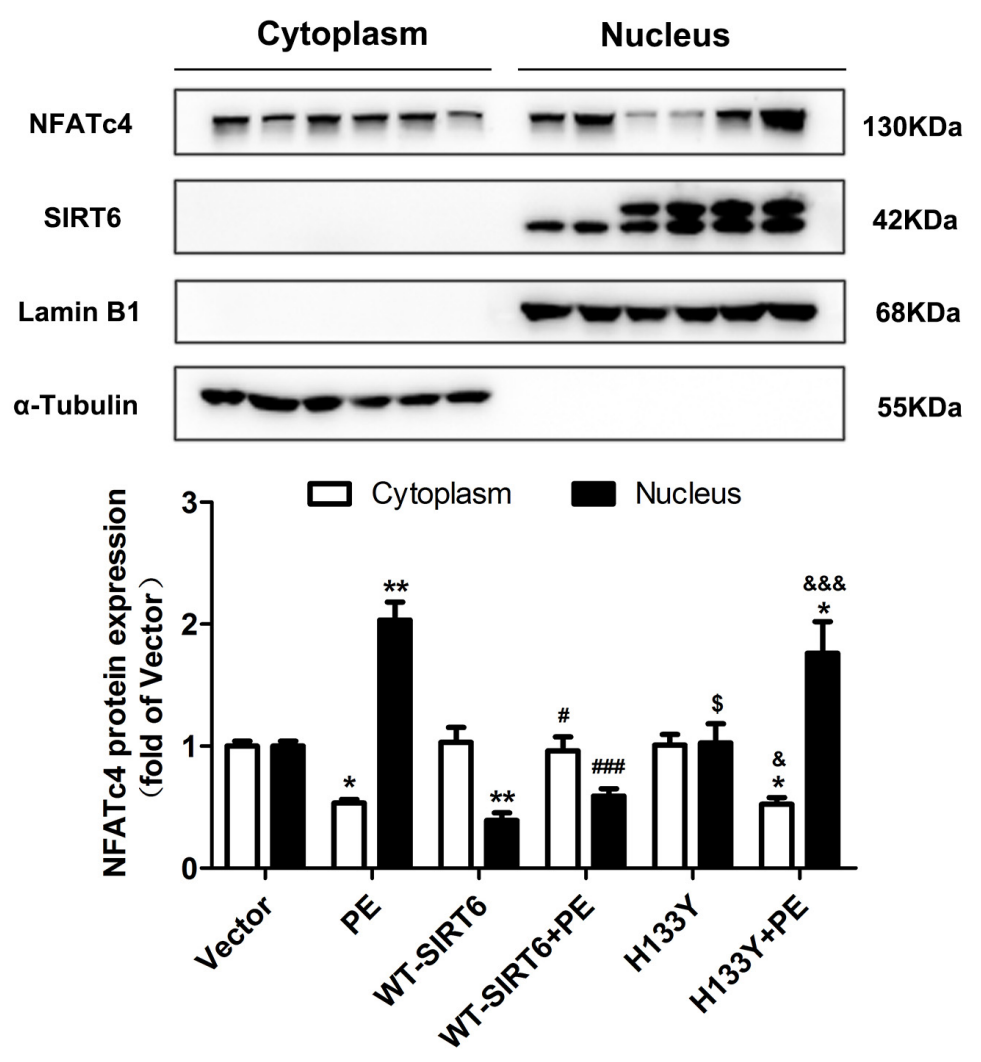

B
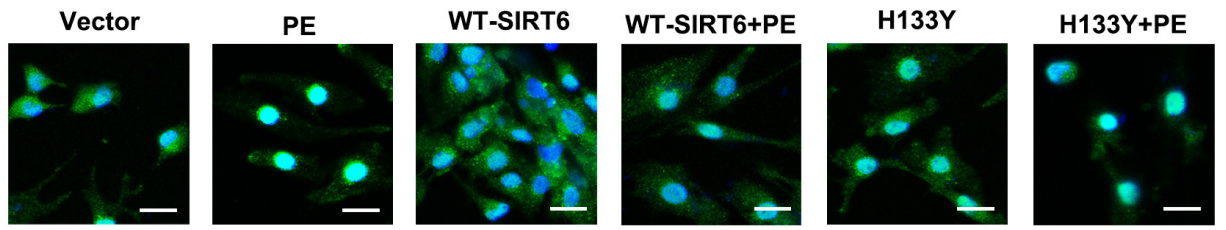

FIGURE 5 | SIRT6 prevented PE-induced nuclear translocation of NFATc4 by interfering with calcineurin-NFATc4 interaction. NRCMs were transiently transfected with plasmid encoding the WT-SIRT6 and mutant of SIRT6 (H133Y) for $48 \mathrm{~h}$ followed by incubation with PE (100 $\mu$ M for $12 \mathrm{~h}$ ). (A) The protein expression of NFATc4 in the nucleus and cytoplasm was measured by Western blotting. (B) The presence of shuttling between the nucleus and cytoplasm of NFATc4 was observed by IF microscopy. The results were normalized to those of $\alpha$-tubulin/LaminB1. The data were presented as mean $\pm \mathrm{SEM}$. ${ }^{*} P<0.05,{ }^{*} P<0.01$ vs. Vector. ${ }^{*} P<0.05$, \#\#\# $P<0.001$ vs. PE. ${ }^{\$} P<0.05$ vs. WT-SIRT6. ${ }^{\&} P<0.05$, ${ }^{\& \& \&} P<0.001$ vs. WT-SIRT6 + PE. $n=4$.

cytoplasm to nucleus. Since the above results indicate that SIRT6 inhibits NFATc4 dephosphorylation, we further investigated whether or not the nuclear accumulation of NFATc4 is affected by SIRT6 overexpression. As shown by the Western blotting data, PE treatment augmented the expression of NFATc4 in the nuclear fractions of cardiomyocytes, but diminished that in the cytoplasmic fractions (Figure 5A). Overexpression of WT-SIRT6 significantly repressed the nuclear expression of NFATc4 in the presence or absence of PE, without affecting the cytoplasmic expression (Figure 5A). However, H133Y itself did not alter NFATc4 expression in either the nuclear fraction or the cytoplasmic fraction. Additionally, H133Y did not influence the effect of PE (Figure 5A). Results of the immunofluorescence experiments further confirmed that WT-SIRT6 but not
H133Y prevented PE-facilitated NFATc4 occupancy in nucleus (Figure 5B).

\section{SIRT6 Suppressed NFATc4 Dephosphorylation and Nuclear Accumulation via Deacetylating It}

The dephosphorylation and translocation of NFATc4 relies on the activation of CaN. However, the above results indicated that WT-SIRT6 could not change the expression of CaN. This finding raises the hypothesis that SIRT6 might alter the kinase which enhances the phosphorylation of NFATc4 to prevent NFATc4 activation. To explore this hypothesis, the expression and phosphorylation of $\mathrm{p} 38$ were investigated. As shown in Supplementary Figure S1, overexpression of SIRT6 did not affect either the phosphorylation or the total expression of $\mathrm{p} 38$, 


\section{A}

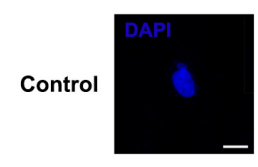

PE

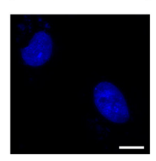

C

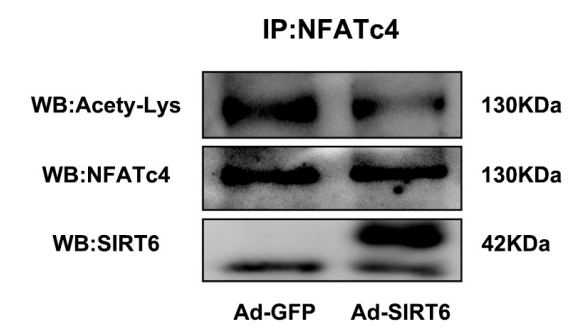

D
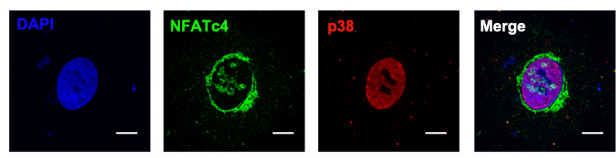

E
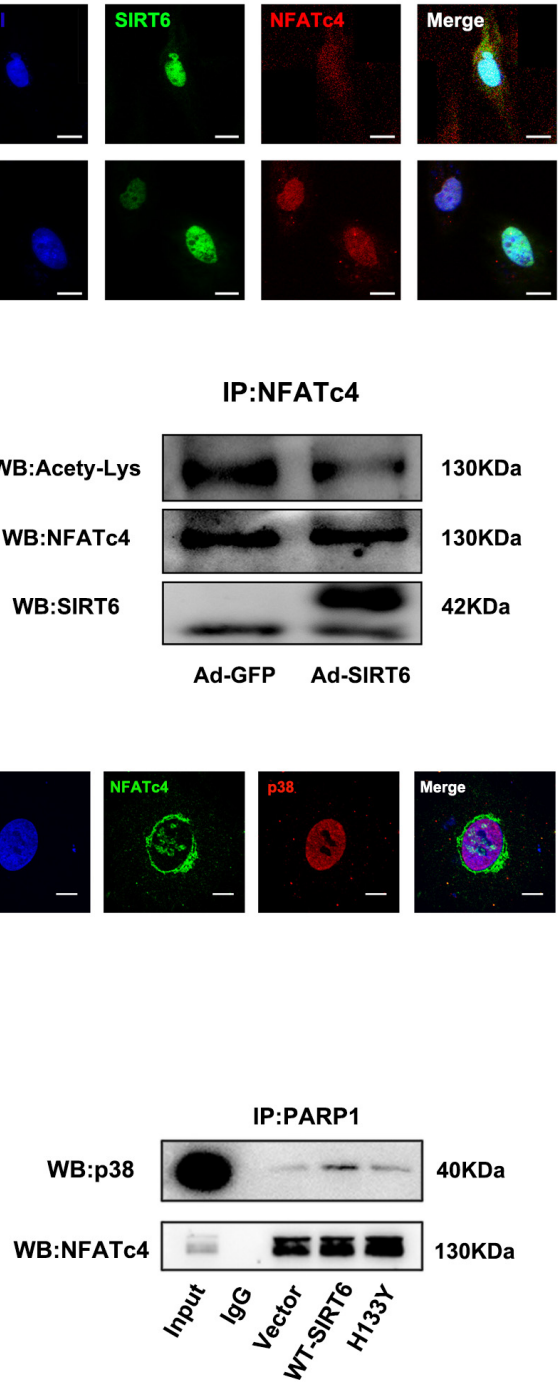

B

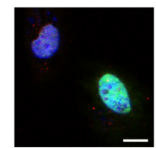

SIRT6

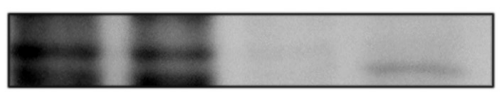

$42 \mathrm{KDa}$

NFATc4

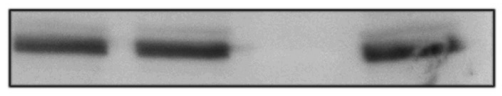

$130 \mathrm{KDa}$
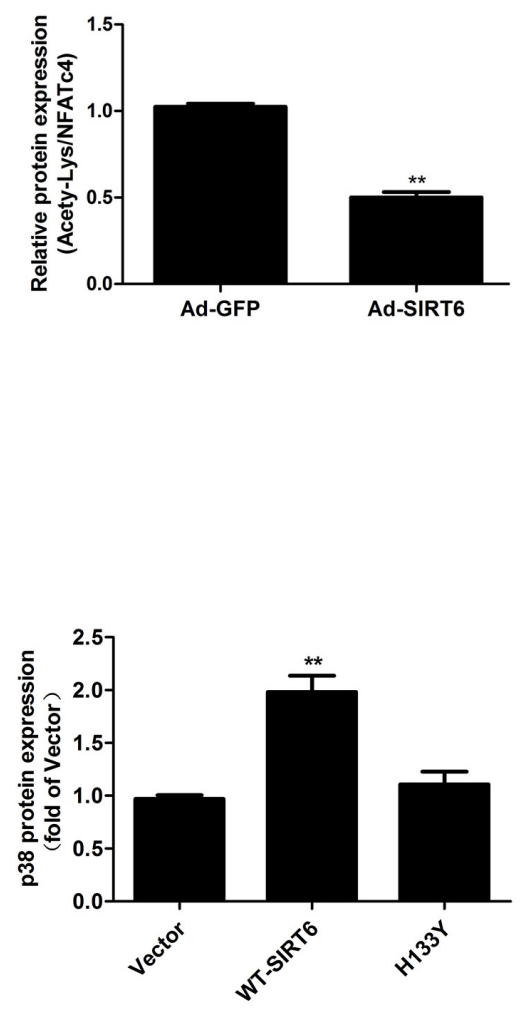

FIGURE 6 | SIRT6 interacted with NFATc4 and deacetylated NFATc4 in NRCMs. (A) The intracellular colocalization of SIRT6 (Green) and NFATc4 (Red) in NRCMs treated with $100 \mu \mathrm{M}$ PE for $12 \mathrm{~h}$. (B) Co-immunoprecipitation shows SIRT6 interact with NFATc4. (C) The acetylated level of NFATc4 was detected in NRCMs infected with or without Ad-SIRT6. (D) The intracellular colocalization of NFATc4 (Green) and p38 (Red) in NRCMs. (E) Co-immunoprecipitation shows p38 interact with NFATc4 after SIRT6 and H133Y overexpression. The data were presented as mean \pm SEM. ${ }^{*} P<0.01$ vs. Ad-GFP. $n=4$.

thus eliminating the possibility that SIRT6 enhances NFATc4 dephosphorylation via activating its kinase p38.

Interestingly, the results from immunofluorescence and CoIP experiments demonstrated that there was interaction and co-localization between SIRT6 and NFATc4 (Figures 6A,B). In addition, SIRT6 overexpression reduced the acetylation level of NFATc4 (Figure 6C), suggesting that SIRT6 probably deacetylates NFATc4. These observations raise the hypothesis that NFATc4 might interact with SIRT6 and undergo deacetylation by SIRT6 in the nucleus, leading to a structural change of NFATc4, and facilitating its interaction with p38, finally enhancing its phosphorylation by $\mathrm{p} 38$ and nuclear export. Because of technical limitation, we failed to directly measure the changes of crystal structure of NFATc4. Instead, we examined the changes of interaction between NFATc4 and p38 after SIRT6 overexpression. The results showed that NFATc4 co-localized and interacted with p38 (Figures 6D,E), and that this interaction was enhanced by overexpression of SIRT6, but not H133Y (Figure 6E), supporting the above hypothesis.

\section{Suppression of NFATc4 Contributed to the Anti-hypertrophic Effect of SIRT6}

To elucidate that suppression of NFATc4 dephosphorylation and nuclear occupancy is involved in the anti-hypertrophic effect of SIRT6, NRCMs were transfected with WT-NFATc4 and WT-SIRT6, or treated with PE, alone or in combination (Figures 7A,B and Supplementary Figure S3B). Overexpression 
A
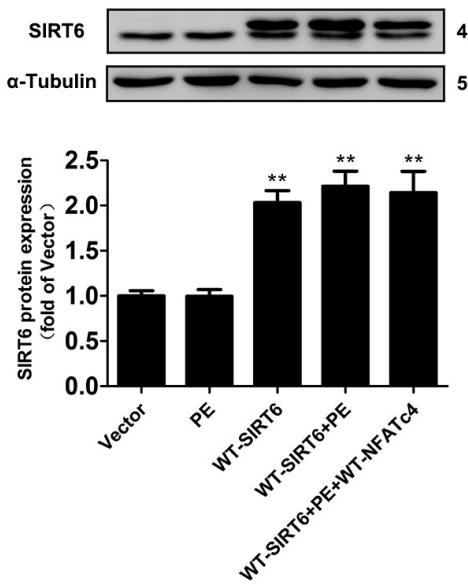

D
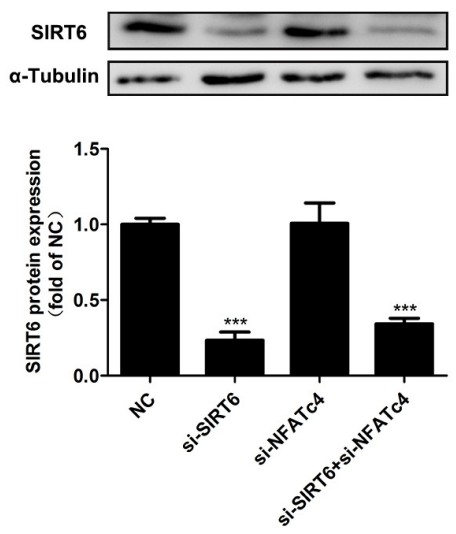

B
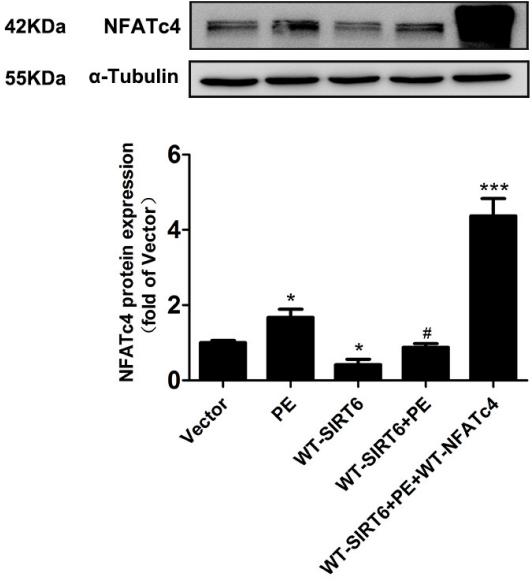

$\mathbf{E}$
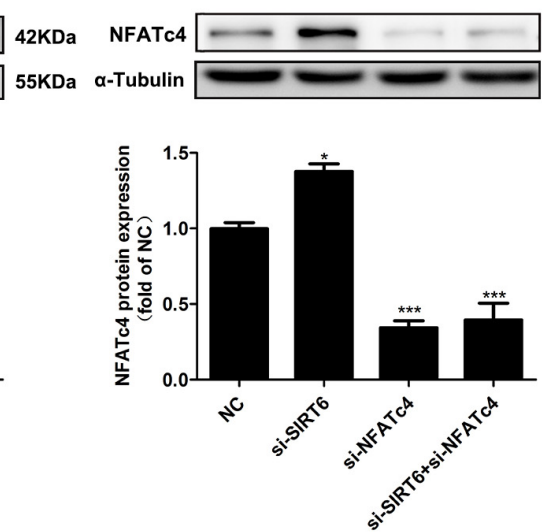

C
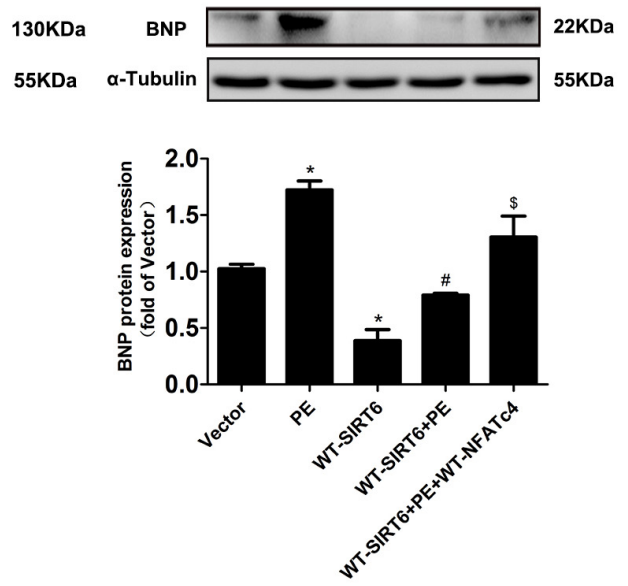

$\mathbf{F}$
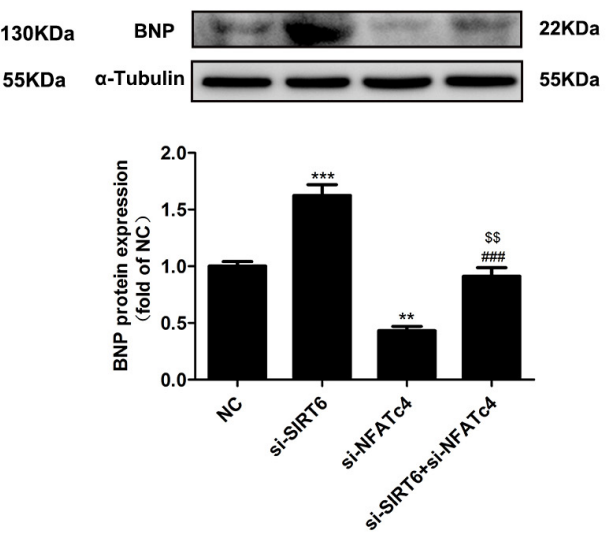

FIGURE 7 | NFATc4 participated in the anti-hypertrophic effect of SIRT6. (A-C) Western blot analysis of the protein expression of SIRT6, NFATC4, and BNP in NRCMs transfected with or without WT-NFATc4, WT-SIRT6, and treated with $100 \mu \mathrm{M}$ PE for $12 \mathrm{~h}$. (D-F) The protein expression of SIRT6, NFATc4, and BNP in NRCMs transfected with siRNAs of SIRT6, NFATC4, or negative control (NC) for $48 \mathrm{~h}$. The data were presented as mean \pm SEM. ${ }^{*} P<0.05$, ${ }^{* *} P<0.01$,

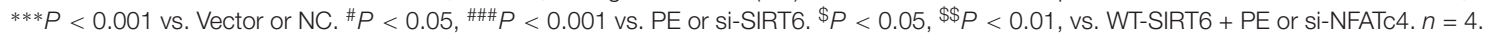

of NFATc4 significantly reversed the effect of SIRT6 on suppressing PE-induced upregulation of BNP (Figure 7C). In addition, endogenous NFATc4 and SIRT6 were knocked down by RNA interference in NRCMs (Figures 7D,E). Knockdown of NFATc4 recovered SIRT6 silencing-facilitated expression of BNP (Figure 7F). These observations suggest that suppression of NFATc4 contributes to the cardioprotective effect of SIRT6.

\section{DISCUSSION}

It is well established that activation of $\mathrm{Ca}^{2+}{ }_{-\mathrm{CaN}-N F A T c 4}$ signaling pathway is associated with mechanical or agonistinduced cardiac hypertrophy (Molkentin et al., 1998; Bernt et al., 2016; Zhao et al., 2016; Lu et al., 2017; Parra and Rothermel, 2017; van Middendorp et al., 2017). In response to myocyte stretch or increased workloads of the heart, or stimuli by a variety of humoral factors including PE, angiotensin II, and endothelin-1, the elevation in intracellular $\mathrm{Ca}^{2+}$ initiates the activation of $\mathrm{CaN}$, a ubiquitously expressed serine/threonine phosphatase, leading to dephosphorylation, and nuclear translocation of NFATc4, thereby facilitating its binding to DNA and interaction with other transcription factors, subsequently enhancing the expression of targeted hypertrophic genes (Molkentin et al., 1998; Coleman et al., 2015; Bernt et al., 2016; Zhao et al., 2016; Lu et al., 2017; Parra and Rothermel, 2017). In line with these findings, the present study demonstrated that expressions of NFATc4 and CaN were increased, whereas the phosphorylation level of NFATc4 was decreased in PE-induced in vitro cardiomyocyte hypertrophy model and AAC-induced in vivo cardiac hypertrophy model (Figures 1, 2).

The major novel finding of the present study is that SIRT6 acts as a negative regulator of CaN-NFATc4 signaling pathway in cardiomyocyte hypertrophy. As a promising therapeutic target for cardiovascular diseases (Vitiello et al., 2017), SIRT6 demonstrates potent anti-hypertrophic effect both in vivo and 
in vitro (Cai et al., 2012; Sundaresan et al., 2012; Yu et al., 2013; Lu et al., 2016; Shen et al., 2016; Zhang et al., 2016). The decrease in deacetylase activity of SIRT6 might contribute to the development of cardiac hypertrophy (Cai et al., 2012; Sundaresan et al., 2012; Yu et al., 2013; Lu et al., 2016; Shen et al., 2016; Zhang et al., 2016). Our study reveals that this anti-hypertrophic effect of SIRT6 involves suppression of CaNNFATc4 signaling pathway. This conclusion is supported by the following observations: (1) Infection of Ad-SIRT6 or transfection of WT-SIRT6 plasmid reversed PE-induced upregulation and dephosphorylation of NFATc4, coordinating with the changes of cardiomyocyte surface area and hypertrophic gene BNP expression (Figures 3, 4); (2) SIRT6 overexpression inhibited PE-induced NFATc4 nuclear shuttling (Figure 5); (3) The effect of SIRT6 on repressing BNP expression was reversed by NFATc4 replenishment (Figures 7A-C); (4) SIRT6 deficiencymediated BNP upregulation was recovered by NFATc4 silencing (Figures 7D-F). It is important to note that the effect of SIRT6 on inhibiting NFATc4 is dependent on its deacetylase activity, taken into account that the SIRT6 mutant H133Y lost the capability to suppress NFATc4 expression, dephosphorylation, and nuclear transport (Figures 4, 5). However, the present study indicates that SIRT6 did not alter protein expression level of $\mathrm{CaN}$, although that was shown an elevation in PE-treated cardiomyocytes and in hearts of AAC rats (Figures 1E, $\mathbf{2 H}$, 3G, 4G).

According to our observations, SIRT6 regulates NFATc4 by at least two ways. First, SIRT6 restrains NFATc4 expression at the transcriptional/post-transcriptional level, considering that overexpression of SIRT6 significantly decreased the mRNA and protein expression level of NFATc4 in the presence or absence of PE (Figures 3C,D, 4D,E). The mechanism by which SIRT6 suppresses NFATc4 expression remains unclear. As a class III histone lysine deacetylase, SIRT6 deacetylates the histones H3K9 and $\mathrm{H} 3 \mathrm{~K} 56$ to modify chromatin and regulate the transcription of a variety of genes (Wang et al., 2016; Agerholm et al., 2018; Wood et al., 2018). Accumulating evidences show that SIRT6 can transcriptionally regulate the expressions of Txnip (Qin et al., 2018), PDK4 (Khan et al., 2018), NF-кB target genes (Yu et al., 2013), and insulin-like growth factor signaling-related genes (Sundaresan et al., 2012) by catalyzing the deacetylation of H3K9 and/or $\mathrm{H} 3 \mathrm{~K} 56$ at their respective promoter regions. Therefore, it is possible that SIRT6 deacetylates potential histones at NFATc4 promoter region and thereby represses the transcription of NFATc4. This hypothesis is supported by the present results that the deacetylation activity of SIRT6 was required to attenuate NFATc4 expression (Figures 4D,E). In addition, the expression of NFATc4 is also repressed by microRNAs such as miR-133a and miR-29a-3p (Li et al., 2010, 2016b). Taken into considerations that SIRT6 plays an important role in regulating microRNAs (Fan et al., 2016; Kugel et al., 2016), it is hypothesized that SIRT6 suppresses NFATc4 expression via regulating miR-133a and miR$29 a-3 p$. In order to validate this hypothesis, we have examined the expression of miR-133a and miR-29a-3p in SIRT6-overexpressed cells. The results showed that SIRT6 overexpression significantly increased the expression of miR-133a, but did not affect miR-29a3 p (Supplementary Figure S2). Thus, it is most likely that SIRT6 suppresses NFATc4 expression via augmenting the expression of miR-133a.

Second, SIRT6 inhibits NFATc4 activation by augmenting its phosphorylation level and preventing its nuclear accumulation. This conclusion is supported by the observations that the phosphorylated level of NFATc4 was increased (Figures 3D,E, 4E,F), but the nuclear expression was reduced after SIRT6 overexpression (Figure 5). The phosphorylation of NFATc4 is a balanced action between the phosphatase $\mathrm{CaN}$ (also known as type-2B phosphatase, PP2B) and the potential kinases. Since our results demonstrated that SIRT6 did not influence the expression of CaN (Figures 3G, 4G), it seems most likely that SIRT6 might have an impact on the kinases participating in NFATc4 phosphorylation. The mitogen-activated protein kinases (MAPK) are considered to be crucial co-regulators of NFAT family (Coleman et al., 2015). The MAPK signaling pathway is generally subclassified into three main branches consisting of p38, c-Jun N-terminal kinases (JNKs), and extracellular signalregulated kinases (ERKs) (Kasuya et al., 2018). Among these three sub-families, JNK and p38 are capable of phosphorylating NFATs directly in their $\mathrm{N}$-terminal regulatory domains, resulting in net inhibition of nuclear occupancy. JNK is responsible for phosphorylating NFATc1, NFATc2, and NFATc3, but not NFATc4 (Chow et al., 1997; Porter et al., 2000; Yang et al., 2002); by contrast, p38 is shown to directly phosphorylate NFATc1, NFATc2, and NFATc4, rather than NFATc3. Inhibition of p38 activates CaN-NFAT signaling to promote hypertrophic cardiomyopathy (Gomez del Arco et al., 2000; Porter et al., 2000; Yang et al., 2002). These studies elicit the hypothesis that SIRT6 regulates NFATc4 phosphorylation by activating p38. However, our results indicated that SIRT6 overexpression did not alter p38 expression or phosphorylation (Supplementary Figure S1), thus excluding the possibility that SIRT6 regulates NFATc4 phosphorylation and nuclear accumulation via p38 activation. Surprisingly, NFATfc4 was shown to co-localize and interact with SIRT6, especially when it was activated and transported to the nucleus by PE treatment (Figure 6). Therefore, it is possible that NFATc4 might interact with SIRT6 and undergo deacetylation by SIRT6 in the nucleus, leading to a structural change of NFATc4 and facilitating its interaction with p38, finally enhancing its phosphorylation and nuclear export. This possibility was supported by the observations that the interaction between NFATc4 and p38 was increased by overexpression of SIRT6 rather than $\mathrm{H} 133 \mathrm{Y}$, in the meantime when NFATc4 acetylation was abrogated (Figures 6C,E).

\section{CONCLUSION}

In conclusion, the present study identifies SIRT6 as a negative regulator of NFATc4 in cardiomyocyte hypertrophy. SIRT6 can repress NFATc4 expression and prevent its dephosphorylation and nuclear accumulation, dependent on its deacetylase activity. These findings provide more evidences of the anti-hypertrophic effect of SIRT6, and further suggest that targeting activation of SIRT6 might be promising strategies for treating cardiac hypertrophy and heart failure. 


\section{AUTHOR CONTRIBUTIONS}

ZL, XZ, ZL, and PL designed and conducted the study, analyzed the data, and prepared the manuscript. ZG and JL performed the animal experiments. PW and YZ collected and interpreted the data.

\section{FUNDING}

This research was supported by grants from the National Natural Science Foundation of China (Grant Nos. 81473205 and 81673433), National Engineering and Technology Research Center for New Drug Druggability Evaluation (Seed Program of Guangdong Province), Major Project of Guangdong

\section{REFERENCES}

Agerholm, M., Dall, M., Jensen, B. A. H., Prats, C., Madsen, S., Basse, A. L., et al. (2018). Perturbations of $\mathrm{NAD}(+)$ salvage systems impact mitochondrial function and energy homeostasis in mouse myoblasts and intact skeletal muscle. Am. J. Physiol. Endocrinol. Metab. 314, E377-E395. doi: 10.1152/ajpendo.00213

Appert-Collin, A., Cotecchia, S., Nenniger-Tosato, M., Pedrazzini, T., and Diviani, D. (2007). The A-kinase anchoring protein (AKAP)Lbc-signaling complex mediates alphal adrenergic receptor-induced cardiomyocyte hypertrophy. Proc. Natl. Acad. Sci. U.S.A. 104, 10140-10145. doi: 10.1073/pnas.0701099104

Bernt, A., Rangrez, A. Y., Eden, M., Jungmann, A., Katz, S., Rohr, C., et al. (2016). Sumoylation-independent activation of Calcineurin-NFAT-signaling via SUMO2 mediates cardiomyocyte hypertrophy. Sci. Rep. 6:35758. doi: 10. 1038/srep35758

Cai, Y., Yu, S. S., Chen, S. R., Pi, R. B., Gao, S., Li, H., et al. (2012). Nmnat2 protects cardiomyocytes from hypertrophy via activation of SIRT6. FEBS Lett. 586, 866-874. doi: 10.1016/j.febslet.2012.02.014

Chow, C. W., Rincon, M., Cavanagh, J., Dickens, M., and Davis, R. J. (1997). Nuclear accumulation of NFAT4 opposed by the JNK signal transduction pathway. Science 278, 1638-1641. doi: 10.1126/science.278.5343.1638

Coleman, M. A., Sasi, S. P., Onufrak, J., Natarajan, M., Manickam, K., Schwab, J., et al. (2015). Low-dose radiation affects cardiac physiology: gene networks and molecular signaling in cardiomyocytes. Am. J. Physiol. Heart Circ. Physiol. 309, H1947-H1963. doi: 10.1152/ajpheart.00050.2015

Cotecchia, S., Del Vescovo, C. D., Colella, M., Caso, S., and Diviani, D. (2015). The alpha1-adrenergic receptors in cardiac hypertrophy: signaling mechanisms and functional implications. Cell Signal. 27, 1984-1993. doi: 10.1016/j.cellsig.2015. 06.009

Crabtree, G. R. (1999). Generic signals and specific outcomes: signaling through Ca2+, calcineurin, and NF-AT. Cell 96, 611-614. doi: 10.1016/S0092-8674(00) 80571- 1

Fan, X., Chen, J., Shi, D., Jia, J., He, J., Li, L., et al. (2016). The role and mechanisms of action of SIRT6 in the suppression of postoperative epidural scar formation. Int. J. Mol. Med. 37, 1337-1344. doi: 10.3892/ijmm.2016.2522

Frey, N., Katus, H. A., Olson, E. N., and Hill, J. A. (2004). Hypertrophy of the heart: a new therapeutic target? Circulation 109, 1580-1589. doi: 10.1161/01. CIR.0000120390.68287.BB

Gomez del Arco, P., Martinez-Martinez, S., Maldonado, J. L., Ortega-Perez, I., and Redondo, J. M. (2000). A role for the p38 MAP kinase pathway in the nuclear shuttling of NFATp. J. Biol. Chem. 275, 13872-13878. doi: 10.1074/jbc.275.18. 13872

Govindsamy, A., Naidoo, S., and Cerf, M. E. (2018). Cardiac development and transcription factors: insulin signalling, insulin resistance, and intrauterine nutritional programming of cardiovascular disease. J. Nutr. Metab. 2018:8547976. doi: 10.1155/2018/8547976

Guo, Z., Liao, Z., Huang, L., Liu, D., Yin, D., and He, M. (2015). Kaempferol protects cardiomyocytes against anoxia/reoxygenation injury via mitochondrial
Provincial Department of Science and Technology (Grant Nos. 2015B020232009, 2014B020210003, and 2013B090700010), Major Project of Platform Construction Education Department of Guangdong Province (Grant No. 2014GKPT002), Guangzhou Science and Technology Program Project (Grant No. 201604020121), and funding from Guangdong Provincial Key Laboratory of Construction Foundation (Grant No. 2017B030314030).

\section{SUPPLEMENTARY MATERIAL}

The Supplementary Material for this article can be found online at: https://www.frontiersin.org/articles/10.3389/fphar. 2018.01519/full\#supplementary-material

pathway mediated by SIRT1. Eur. J. Pharmacol. 761, 245-253. doi: 10.1016/j. ejphar.2015.05.056

Guo, Z., Lu, J., Li, J., Wang, P., Li, Z., Zhong, Y., et al. (2018). JMJD3 inhibition protects against isoproterenol-induced cardiac hypertrophy by suppressing beta-MHC expression. Mol. Cell Endocrinol. 477, 1-14. doi: 10.1016/j.mce.2018. 05.009

Heineke, J., and Molkentin, J. D. (2006). Regulation of cardiac hypertrophy by intracellular signalling pathways. Nat. Rev. Mol. Cell Biol. 7, 589-600. doi: 10.1038/nrm1983

Horsley, V., and Pavlath, G. K. (2002). NFAT: ubiquitous regulator of cell differentiation and adaptation. J. Cell Biol. 156, 771-774. doi: 10.1083/jcb. 200111073

Kasuya, Y., Umezawa, H., and Hatano, M. (2018). Stress-activated protein kinases in spinal cord injury: focus on roles of p38. Int. J. Mol. Sci. 19:E867. doi: 10.3390/ijms 19030867

Khan, D., Sarikhani, M., Dasgupta, S., Maniyadath, B., Pandit, A. S., Mishra, S., et al. (2018). SIRT6 deacetylase transcriptionally regulates glucose metabolism in heart. J. Cell. Physiol. 233, 5478-5489. doi: 10.1002/jcp.26434

Kugel, S., Sebastian, C., Fitamant, J., Ross, K. N., Saha, S. K., Jain, E., et al. (2016). SIRT6 suppresses pancreatic cancer through control of lin28b. Cell 165, 1401-1415. doi: 10.1016/j.cell.2016.04.033

Li, M., He, H. P., Gong, H. Q., Zhang, J., Ma, W. J., Zhou, H., et al. (2016a). NFATc4 and myocardin synergistically up-regulate the expression of LTCC alpha1C in ET-1-induced cardiomyocyte hypertrophy. Life Sci. 155, 11-20. doi: 10.1016/j.lfs.2016.05.007

Li, M., Wang, N., Zhang, J., He, H. P., Gong, H. Q., Zhang, R., et al. (2016b). MicroRNA-29a-3p attenuates ET-1-induced hypertrophic responses in H9c2 cardiomyocytes. Gene 585, 44-50. doi: 10.1016/j.gene.2016.03.015

Li, Q., Lin, X., Yang, X., and Chang, J. (2010). NFATc4 is negatively regulated in miR-133a-mediated cardiomyocyte hypertrophic repression. Am. J. Physiol. Heart Circ. Physiol. 298, H1340-H1347. doi: 10.1152/ajpheart.00592. 2009

Li, S., Pan, Y., Ke, R., Xie, X., Zhai, C., Shi, W., et al. (2017). Inhibition of phosphodiesterase-5 suppresses calcineurin/NFAT- mediated TRPC6 expression in pulmonary artery smooth muscle cells. Sci. Rep. 7:6088. doi: 10.1038/s41598-017-06350-5

Liu, X. P., Gao, H., Huang, X. Y., Chen, Y. F., Feng, X. J., He, Y. H., et al. (2015). Peroxisome proliferator-activated receptor gamma coactivator 1 alpha protects cardiomyocytes from hypertrophy by suppressing calcineurin-nuclear factor of activated T cells c4 signaling pathway. Transl. Res. 166, 459.e-473.e. doi: 10.1016/j.trsl.2015.06.003

Lu, J., Sun, D., Liu, Z., Li, M., Hong, H., Liu, C., et al. (2016). SIRT6 suppresses isoproterenol-induced cardiac hypertrophy through activation of autophagy. Transl. Res. 172, 96.e-112.e. doi: 10.1016/j.trsl.2016.03.002

Lu, Y., Zhao, M., Liu, J. J., He, X., Yu, X. J., Liu, L. Z., et al. (2017). Longterm administration of pyridostigmine attenuates pressure overload-induced cardiac hypertrophy by inhibiting calcineurin signalling. J. Cell Mol. Med. 21, 2106-2116. doi: 10.1111/jcmm.13133 
Mendes, K. L., Lelis, D. F., and Santos, S. H. S. (2017). Nuclear sirtuins and inflammatory signaling pathways. Cytokine Growth. Factor Rev. 38, 98-105. doi: 10.1016/j.cytogfr.2017.11.001

Molkentin, J. D., Lu, J. R., Antos, C. L., Markham, B., Richardson, J., Robbins, J., et al. (1998). A calcineurin-dependent transcriptional pathway for cardiac hypertrophy. Cell 93, 215-228. doi: 10.1016/S0092-8674(00)81573-1

Parra, V., and Rothermel, B. A. (2017). Calcineurin signaling in the heart: the importance of time and place. J. Mol. Cell Cardiol. 103, 121-136. doi: 10.1016/j. yjmcc.2016.12.006

Porter, C. M., Havens, M. A., and Clipstone, N. A. (2000). Identification of amino acid residues and protein kinases involved in the regulation of NFATc subcellular localization. J. Biol. Chem. 275, 3543-3551. doi: 10.1074/jbc.275.5. 3543

Qin, K., Zhang, N., Zhang, Z., Nipper, M., Zhu, Z., Leighton, J., et al. (2018). SIRT6mediated transcriptional suppression of Txnip is critical for pancreatic beta cell function and survival in mice. Diabetologia 61, 906-918. doi: 10.1007/s00125017-4542-6

Rohini, A., Agrawal, N., Koyani, C. N., and Singh, R. (2010). Molecular targets and regulators of cardiac hypertrophy. Pharmacol. Res. 61, 269-280. doi: 10.1016/j. phrs.2009.11.012

Sharma, A., Halu, A., Decano, J. L., Padi, M., Liu, Y. Y., Prasad, R. B., et al. (2018). Controllability in an islet specific regulatory network identifies the transcriptional factor NFATC4, which regulates type 2 diabetes associated genes. NPJ Syst. Biol. Appl. 4:25. doi: 10.1038/s41540-018-0057-0

Shen, P., Feng, X., Zhang, X., Huang, X., Liu, S., Lu, X., et al. (2016). SIRT6 suppresses phenylephrine-induced cardiomyocyte hypertrophy though inhibiting p300. J. Pharmacol. Sci. 132, 31-40. doi: 10.1016/j.jphs.2016.03.013

Sosnowska, B., Mazidi, M., Penson, P., Gluba-Brzozka, A., Rysz, J., and Banach, M. (2017). The sirtuin family members SIRT1, SIRT3 and SIRT6: their role in vascular biology and atherogenesis. Atherosclerosis 265, 275-282. doi: 10.1016/ j.atherosclerosis.2017.08.027

Sundaresan, N. R., Vasudevan, P., Zhong, L., Kim, G., Samant, S., Parekh, V., et al. (2012). The sirtuin SIRT6 blocks IGF-Akt signaling and development of cardiac hypertrophy by targeting c-Jun. Nat. Med. 18, 1643-1650. doi: 10.1038/nm.2961

Tasselli, L., Zheng, W., and Chua, K. F. (2017). SIRT6: novel mechanisms and links to aging and disease. Trends Endocrinol. Metab. 28, 168-185. doi: 10.1016/j.tem. 2016.10.002

van Middendorp, L. B., Kuiper, M., Munts, C., Wouters, P., Maessen, J. G., van Nieuwenhoven, F. A., et al. (2017). Local microRNA-133a downregulation is associated with hypertrophy in the dyssynchronous heart. ESC Heart Fail. 4, 241-251. doi: 10.1002/ehf2.12154

Vitiello, M., Zullo, A., Servillo, L., Mancini, F. P., Borriello, A., Giovane, A., et al. (2017). Multiple pathways of SIRT6 at the crossroads in the control of longevity, cancer, and cardiovascular diseases. Ageing Res. Rev. 35, 301-311. doi: 10.1016/j.arr.2016.10.008

Wang, W. W., Zeng, Y., Wu, B., Deiters, A., and Liu, W. R. (2016). A chemical biology approach to reveal sirt6-targeted histone h3 sites in nucleosomes. ACS Chem. Biol. 11, 1973-1981. doi: 10.1021/acschembio.6b0 0243

Wang, X. D., Qi, X. X., Bian, Y., Chen, X. D., Zhang, Y. Y., and Fang, Z. Y. (2017). Huangqi danshen decoction attenuates isoproterenol-induced myocardial remodeling via STIM1, TRPC1, CaN and NFATc3 pathways in rats. Zhongguo Zhong Yao Za Zhi 42, 2738-2743. doi: 10.19540/j.cnki.cjcmm. 20170609.013

Wood, M., Rymarchyk, S., Zheng, S., and Cen, Y. (2018). Trichostatin a inhibits deacetylation of histone $\mathrm{H} 3$ and p53 by SIRT6. Arch. Biochem. Biophys. 638, 8-17. doi: 10.1016/j.abb.2017.12.009

Yang, T. T., Xiong, Q., Enslen, H., Davis, R. J., and Chow, C. W. (2002). Phosphorylation of NFATc4 by p38 mitogen-activated protein kinases. Mol. Cell. Biol. 22, 3892-3904. doi: 10.1128/MCB.22.11.3892-3904.2002

Yu, S. S., Cai, Y., Ye, J. T., Pi, R. B., Chen, S. R., Liu, P. Q., et al. (2013). Sirtuin 6 protects cardiomyocytes from hypertrophy in vitro via inhibition of NF-kappaB-dependent transcriptional activity. Br. J. Pharmacol. 168, 117-128. doi: 10.1111/j.1476-5381.2012.01903.x

Yuan, Q., Chen, Z., Santulli, G., Gu, L., Yang, Z. G., Yuan, Z. Q., et al. (2014). Functional role of Calstabin2 in age-related cardiac alterations. Sci. Rep. 4:7425. doi: 10.1038/srep07425

Zhang, X., Li, W., Shen, P., Feng, X., Yue, Z., Lu, J., et al. (2016). STAT3 suppression is involved in the protective effect of SIRT6 against cardiomyocyte hypertrophy. J. Cardiovasc. Pharmacol. 68, 204-214. doi: 10.1097/FJC.0000000000000404

Zhang, Y., and Storey, K. B. (2016). Expression of nuclear factor of activated $\mathrm{T}$ cells (NFAT) and downstream muscle-specific proteins in ground squirrel skeletal and heart muscle during hibernation. Mol. Cell. Biochem. 412, 27-40. doi: 10.1007/s11010-015-2605-x

Zhao, Y., Cui, G. M., Zhou, N. N., Li, C., Zhang, Q., Sun, H., et al. (2016). Calpaincalcineurin-nuclear factor signaling and the development of atrial fibrillation in patients with valvular heart disease and diabetes. J. Diabetes Res. 2016, 4639654. doi: 10.1155/2016/4639654

Zhou, S. G., Zhou, S. F., Huang, H. Q., Chen, J. W., Huang, M., and Liu, P. Q. (2006). Proteomic analysis of hypertrophied myocardial protein patterns in renovascularly hypertensive and spontaneously hypertensive rats. J. Proteome Res. 5, 2901-2908. doi: 10.1021/pr0504561

Conflict of Interest Statement: The authors declare that the research was conducted in the absence of any commercial or financial relationships that could be construed as a potential conflict of interest.

Copyright (c) 2019 Li, Zhang, Guo, Zhong, Wang, Li, Li and Liu. This is an open-access article distributed under the terms of the Creative Commons Attribution License (CC BY). The use, distribution or reproduction in other forums is permitted, provided the original author(s) and the copyright owner(s) are credited and that the original publication in this journal is cited, in accordance with accepted academic practice. No use, distribution or reproduction is permitted which does not comply with these terms. 